\title{
In-Service BER Based Estimation of OFDM PAPR and CFO-induced Peak Phase Deviation
}

\author{
Adriana Lipovac, and Ante Mihaljević
}

\begin{abstract}
High Peak-to-Average Power Ratio (PAPR) of the transmitted OFDM signal and the Carrier Frequency Offset (CFO) are well-known major drawbacks of the Orthogonal Frequency-Division Multiplexing (OFDM). Therefore, to avoid the High-Power Amplifier (HPA) inefficient operation with large back-off between the operating input power and its saturation region, specifically with the Long-Term Evolution (LTE) systems downlink, PAPR and CFO must be minimized. Considering that in many practical situations, this task demands (often unavailable) complex test equipment, such as e.g. Vector Signal Analyzer (VSA), in this paper, we develop a simple model for estimating the (residual) PAPR and CFO-induced peak phase deviation, by applying link abstraction, i.e. considering the easymeasurable BER degradation due to HPA non-linearity, as if it were the consequence of the according additive white Gaussian noise (AWGN). Moreover, the classic out-of-service BER testing, which requires network operator to interrupt its revenuegenerating traffic, is substituted by in-service BER estimation from in-phase and quadrature-phase eye diagram closures, measured on live traffic, by means of a simple oscilloscope. The analytical model is verified by the appropriate Monte-Carlo simulations.
\end{abstract}

Index Terms-PAPR, OFDM, LTE, BER.

\section{INTRODUCTION}

Orthogonal Frequency-Division Multiplexing (OFDM) offers high-speed and excellent-performance data transmission over time-dispersive channels [1]. Therefore, it has been extensively used throughout the second consecutive decade, ever since it started to be deployed with the wireless local-area networks, through digital video broadcasting, and finally to the fourth-generation $(4 \mathrm{G})$ mobile communication systems - the Long-Term Evolution (LTE), in particular [2-5].

However, two main imperfections of OFDM systems still remain in focus when the OFDM transmission performance is in question, namely the Carrier Frequency Offset (CFO) that disrupts the orthogonality and so causes the Inter-Carrier Interference (ICI), as well as the excessive Peak-to-Average Power Ratio (PAPR) that is immanent even to the ideal OFDM signal, as its particular subcarriers combine one with e-

Manuscript received September 5, 2018; revised November 26, 2018. Date of publication February 15, 2019

Authors are with the University of Dubrovnik, Croatia (e-mails: adriana.lipovac@unidu.hr, ante.mihaljevic@hotmail.com).

Digital Object Identifier (DOI): 10.24138/jcomss.v15i1.614. ach other either constructively, or destructively [6-7].

On the other hand, at the transmitter, large OFDM signal peaks pass through the High-Power Amplifier (HPA), which therefore is necessary to operate in its linear region, i.e. with large back-off between the operating input power and its value at which the HPA starts entering its saturation region.

Specifically with LTE downlink, some kind of PAPR reduction must be utilized, while in LTE uplink, the SingleCarrier-Frequency-Division Multiple Access (SC-FDMA), characterized by lower PAPR, and the mobile station - User Equipment (UE) operating at much lower power level, both imply that no PAPR reduction needs to be done.

However, as LTE-Advanced enables carrier aggregation in the downlink, even higher PAPR values can be expected, demanding wider linear HPA dynamic range, while the clustered SC-FDMA implies the same in the uplink [3].

With this regard, specifically with LTE-Advanced downlink, quick and simple OFDM PAPR estimation can be of interest. Therefore, in this paper the link abstraction principle is applied on the BER considered to be determined just by the HPA nonlinear distortion, i.e. provided that the channel is noiseless and time-dispersion-free (so that errors occur just due to HPA nonlinearity and the actual PAPR).

Finally, we extend the model to include the BER based estimation of $\mathrm{CFO}$-induced peak phase deviation.

In Section 2, concepts of DPD and PAPR reduction are presented as well as relevant measurement examples. Then, in Section 3, we present the BER based PAPR, as well as CFOinduced peak phase deviation estimation, for the high-Signalto-Noise Ratio (SNR) and multipath-free common AWGN channel that would have produced the same BER value. In Section 4, we review the in-service BER estimation from eye diagram closures measured on live traffic, using an oscilloscope. The analytical model is verified by means of Monte Carlo (MC) simulations, while conclusions are summarized in Section 5 .

\section{HPA NONLINEARITY COMPENSATION}

Linear HPA operation implies low power efficiency:

$$
\eta=\frac{P_{\text {out }}-P_{\text {in }}}{P_{\mathrm{DC}}}
$$


where $P_{\text {out }}, P_{\text {in }}$ and $P_{\mathrm{DC}}$ denote output and input powers, and the power taken from a battery, respectively, Fig.1.

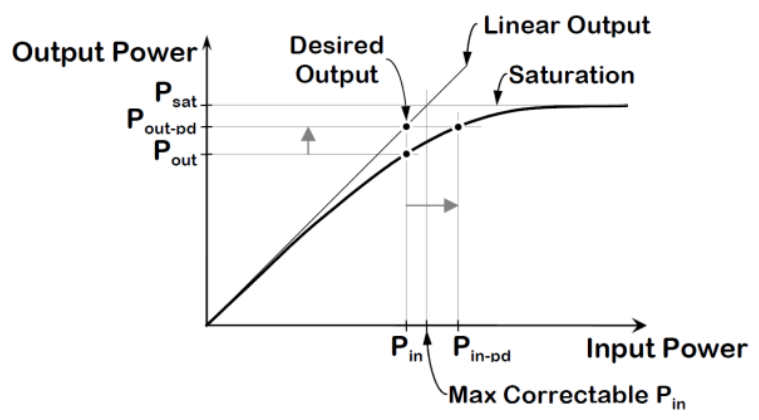

Fig. 1. HPA characteristics

With low $\eta$ in linear regime, typically less than $10 \%$, i.e. with more than $90 \%$ of the DC power dissipated into heat, the HPA operating costs become unacceptable, especially for mobile devices.

However, when an HPA operates in its saturation region, it introduces spectral regrowth that interferes with adjacent channels, Fig. 2.

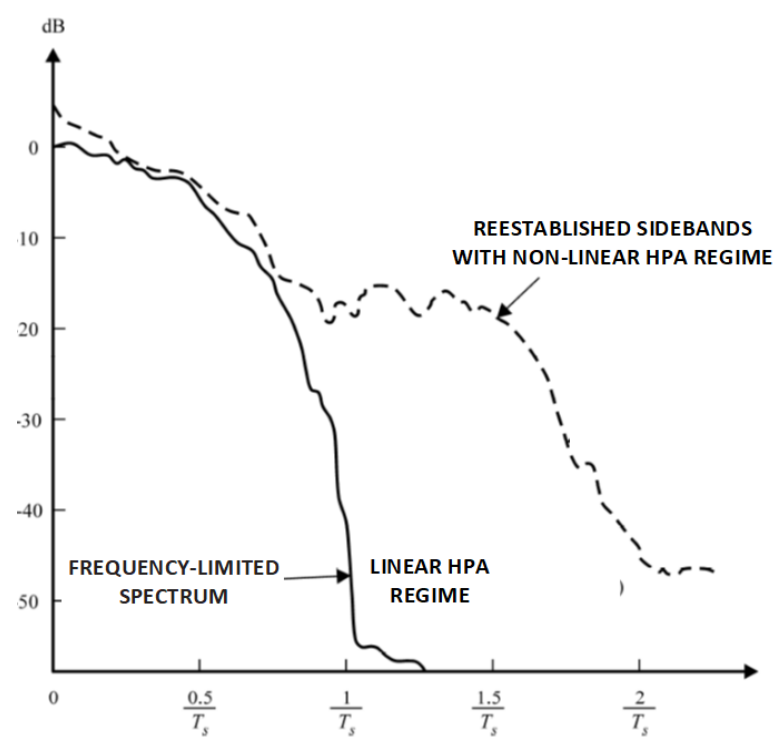

Fig. 2. 4QAM spectrum spreading by HPA

Therefore the signal dynamic range must be accommodated to (mostly) fit the HPAs linear region either by digital predistortion (DPD) or by crest factor reduction (CFR)

Whatever the means to reduce OFDM signal peaks, the benefit is the possibility to increase the allowed average signal power, which implies the higher DC power usage efficiency, i.e. shifting the operating point to the right, and enabling longer distance coverage by a mobile station [1].

The effect on the Error Vector Magnitude (EVM) is presented in Fig. 3.

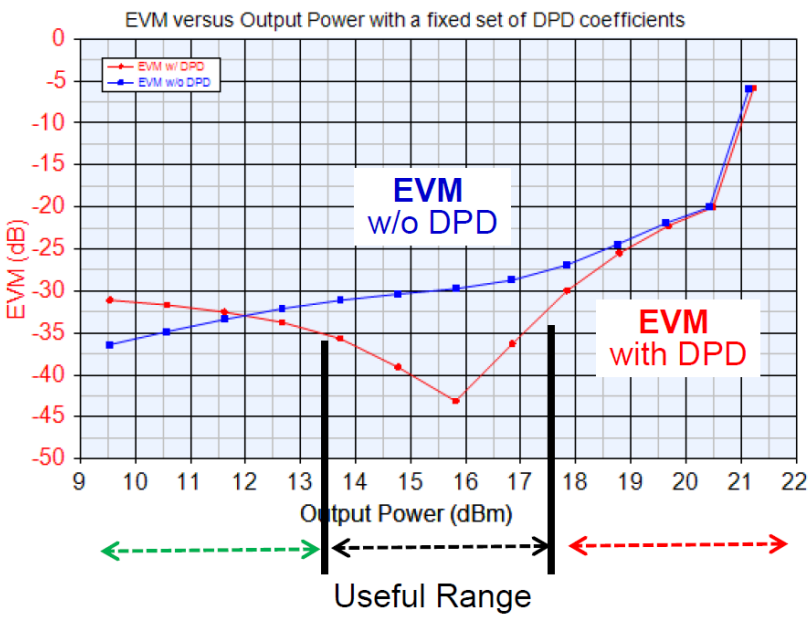

Fig. 3. EVM vs output power with and w/o DPD [3]

Various means to reduce PAPR in OFDM systems have been proposed so far [8-9], ranging from amplitude clipping [9-10], through coding methods [12], as well as the ones that introduce no distortion, with or without side information sent to the receiver [13-15].

\section{TESTING PAPR REDUCTION}

Testing PAPR without and with reduction can discover effectiveness of the applied PAPR reduction algorithm. It can be accomplished in a number of ways.

Specifically, the LTE lab with the state-of-the-art vector signal analysis (VSA) and Error Vector Magnitude (EVM) measurements [16] is the best solution for the analysis with this regard [17-18].

The exemplar PAPR complementary cumulative distribution function $(\mathrm{CCDF})$ curve is presented in Fig. 4, where we can see that the probability that PAPR without CFR gets larger than $8.9 \mathrm{~dB}$ equals $0.1 \%$, which holds already for the PAPR of $7.2 \mathrm{~dB}$, with CFR applied.

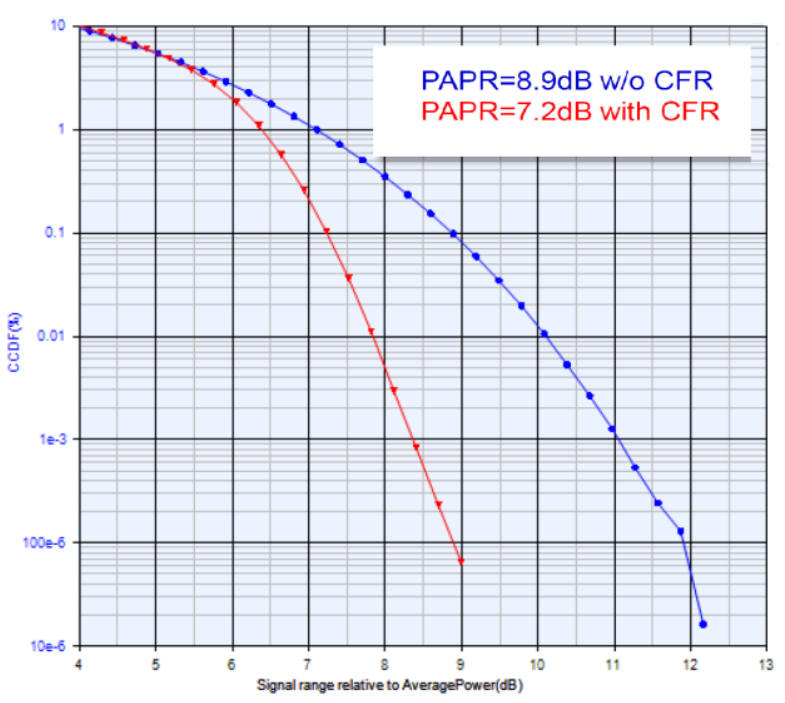

Fig. 4. CCDF of PAPR for with and w/o implementation of CFR in LTEAdvanced with $2 \times 20 \mathrm{MHz}$ contiguous CA [3] 
However, in many practical situations no complex test equipment is available or affordable, so a simple and effective quick (residual) PAPR estimation can be of interest.

Although state-of-the-art wireless systems transmission performance specifications for network operators, such as the LTE ones, are block-error oriented, rather than bit-error based [4], still BER is inevitable performance indicator in research, product design and manufacturing, and is frequently and widely used. So, the idea here is to estimate the postreduction PAPR by means of BER, which ultimately reflects the transmission performance [1].

Thereby, as common BER testing is much easier to accomplish than the VSA, it could be useful to relate PAPR directly to BER targets, rather than the EVM ones, as it follows.

\section{A. BER based PAPR Estimation}

Essentially, the wideband signals' PAPR can be quite large, with the values of up to $13 \mathrm{~dB}$ and more, so CFR can supplement DPD, significantly reducing the necessary backoff from saturated power, and thus increasing the HPA efficiency at no cost of signal degradation.

On this way, the distortion metric due to PAPR reduction is usually expressed in terms of EVM. Accordingly, for the CFR of the LTE-Advanced $20 \mathrm{MHz}$ downlink, the EVM target values for the QPSK, 16QAM and 64QAM are below 10\%, $8 \%$ and $6 \%$, respectively [3].

Let us visualize the relationship among the relevant factors of the PAPR reduction, which enables the HPA operating point (conforming to the average signal power) to be less backed off, where the saturation is simplified as beginning at once, rather than smoothly, Fig. 5. (We consider "smooth" transition (bending) not crucial for the PAPR model, but just the width of the linear dynamic range.)

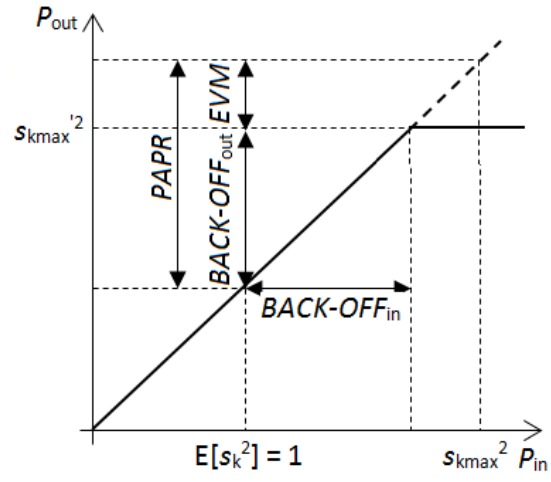

Fig. 5. Relationship between PAPR and EVM, for given back-off [1]

As it can be seen in Fig. 5, ideally, if the whole dynamic range in between the symbol average and peak power, i.e. the actual PAPR value (expressed in decibels), is lesser or equal to the HPA operating point back-off, then there should be no non-linear product at the HPA output.

However, when the maximal-amplitude symbols enter into the negative-back-off zone, then, as already mentioned above, the HPA non-linear distortion occurs, usually expressed as the peak normalized EVM for the largest symbol amplitudes, relative to the ideal (reference) $k$-th symbol power [17]:

$E V M_{k, \max M-\mathrm{QAM}}=\frac{s_{k, \max M-\mathrm{QAM}}-s_{k, \max M-\mathrm{QAM}}^{\prime}}{s_{k, \max M-\mathrm{QAM}}} ; 1 \leq k \leq M(2)$

where $s_{k, \max M-\mathrm{QAM}}^{\prime}$ is the HPA-compressed symbol $s_{k, \max M-\mathrm{QAM}}$.

So, for known HPA operating point back-off, PAPR (in dBs) can be estimated from EVM (in $\mathrm{dBs}$ ) as it follows:

$$
P A P R_{k}=10 \cdot \log \left(10^{\frac{B A C K-O F F}{10}}+10^{\frac{E V M_{k}}{10}}\right) ; 1 \leq k \leq M
$$

Here we consider $B E R$ as being determined just by HPA distortion, as strong signal implies large SNR, while also no time dispersion (and so no multipath fading and the consequent inter-symbol interference) is present due to long enough cyclic prefix.

Now we recall the relationship between the EVM and the HPA-distortion determined BER [17]:

$$
\operatorname{EVM}(B E R)=\sqrt{\frac{3}{M-1}} \cdot \frac{1}{\left[Q^{-1}\left(\frac{B E R \cdot \log _{2} M}{4}\right)\right]}
$$

where $Q^{-1}$ is the inverse of the Gaussian tail function, and MQAM transmission over the AWGN channel is observed [7].

Finally, by substituting (4) into (3), we obtain the PAPR (in $\mathrm{dBs})$ :

$$
P A P R(B E R)=10 \cdot \log \left(10 \frac{B A C K-\text { OFF }}{10}+10 \frac{\sqrt{\frac{3}{M-1}} \cdot\left[Q^{-1}\left(\frac{B E R \cdot \log _{2} M}{4}\right)\right]}{10}\right)
$$

B. BER based Estimation of CFO-induced Peak Phase Deviation

Furthermore, the CFO can create cumulative modulation symbols phase errors, which need to be measured. However, without complex equipment needed to perform constellation analysis, such as Vector Signal Analyzers (VSA) [3] this is not an easy task to do, and simple checking of the achieved CFO compensation, i.e. estimating whether the residual CFOcaused phase error is appropriate, might be very useful in many practical situations ranging from research and product development and manufacturing, to the exploitation phase with LTE network operators.

With this regard, specifically with LTE-Advanced downlink, quick and simple OFDM phase deviation estimation can be of interest. Therefore, in this paper the link abstraction principle is applied on the residual Bit-Error-Rate $(B E R)$ that is considered to be determined just by the CFO, i.e. provided that the channel is noiseless and time-dispersion-free (so that errors occur just due to actual CFO). 
Moreover, recently, it has been shown that the maximal CFO-caused squared phase deviation is linear with the instantaneous (per-OFDM-symbol) PAPR. This implies that any PAPR reduction technique, such as e.g. simple clipping or coding, indirectly suppresses the CFO-induced phase deviation, too [6]. On the other hand this enables determining the CFO-induced phase deviation simply from $B E R$ degradation by applying the link abstraction method.

Thereby, it could be useful to relate the peak CFO-induced phase error to $B E R$, by using the CFO's relationship with PAPR [6] that is expressed by $B E R$ applying link abstraction principle.

As it can be seen in Fig. 6, CFO produces parasitic amplitude and phase modulation, since the $\mathrm{CFO}$-induced error vector $E V_{k}$ modulates the amplitude and the phase of the received nominal (ideal) symbol $\hat{s}_{k}=s_{k} e^{j \varphi_{k}}$ into $\hat{r}_{k, n, \Delta f_{\mathrm{CFO}}}$, where the maximal error $\Delta \Phi_{k}$ of the latter occurs with $E V_{k}$ perpendicular to the nominal $k$-th symbol vector $\hat{s}_{k}$.

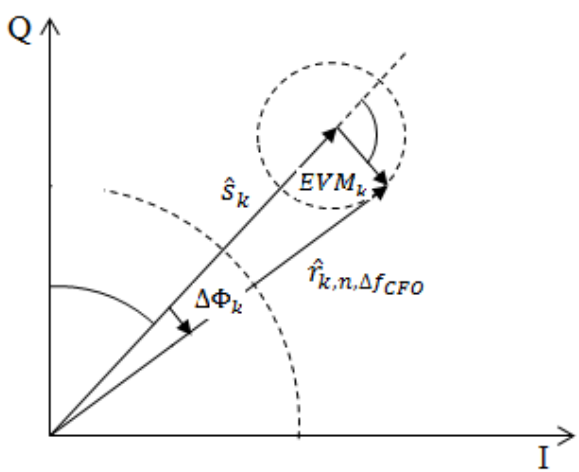

Fig. 6. OFDM CFO-induced modulation symbol phase error model [1]

As it is obvious in Fig. 6 that $\frac{E V M_{k}}{s_{k}}<<1$, the phase deviation can be approximated as:

$\Delta \Phi_{k} \approx \Delta f_{\mathrm{CFO}} M T_{\mathrm{s}} \cdot \frac{E V M_{k}}{s_{k}}=\Delta f_{\mathrm{CFO}} M T_{\mathrm{s}} \cdot \frac{\left|\sum_{\substack{m=1 \\ m \neq k}}^{M} \frac{s_{m} e^{\mathrm{j} \varphi_{m}}}{m-k}\right|}{s_{k}} ; 1 \leq k \leq M$

so that the peak CFO-induced (squared) phase deviation is:

$$
\Delta \Phi_{k}^{2} \approx\left(\Delta f_{\mathrm{CFO}} M T_{\mathrm{s}}\right)^{2} \cdot \frac{\sum_{\substack{m=1 \\ m \neq k}}^{M}\left(\frac{s_{m}}{m-k}\right)^{2}}{s_{k}^{2}}
$$

whose maximal value $\Delta \Phi_{k \max }^{2}$ is shown to be linear with the instantaneous (per-OFDM-symbol) PAPR [1]:

$$
\Delta \Phi_{k \max }^{2} \approx 3 \cdot P A P R_{k} \cdot \frac{\left(\Delta f_{\mathrm{CFO}} M T_{\mathrm{s}}\right)^{2}}{s_{k}^{2}} ; 1 \leq k \leq M
$$

Now, let us relate $\Delta \Phi_{k \max }^{2}$ to $B E R$.

Finally, we substitute (5) into (8) to obtain the relationship between $\Delta \Phi_{k \max }^{2}$ and $B E R$ as it follows:

$$
\begin{aligned}
& \Delta \Phi_{k \max }^{2}(B E R) \approx 30 \cdot \frac{\left(\Delta f_{\mathrm{CFO}} M T_{\mathrm{s}}\right)^{2}}{s_{k}^{2}} . \\
& \cdot \log \left(10 \frac{B A C K-O F F}{10}+10 \frac{\sqrt{\frac{3}{M-1}}\left[\frac{1}{\left[Q^{-1}\left(\frac{B E R \cdot \log _{2} M}{4}\right)\right]}\right.}{10}\right) ; 1 \leq k \leq M
\end{aligned}
$$

\section{PRACTICAL IN-SERVICE BER Estimation}

The well-known BER expression for the M-QAM signal transmission over AWGN channel is [18]:

$$
B E R=\frac{4}{\log _{2} M} \cdot Q\left(\sqrt{\frac{3 \frac{E_{b}}{N_{0}} \cdot \log _{2} M}{M-1}}\right)
$$

where $Q$ denotes the Gaussian tail function, Fig. 7 .

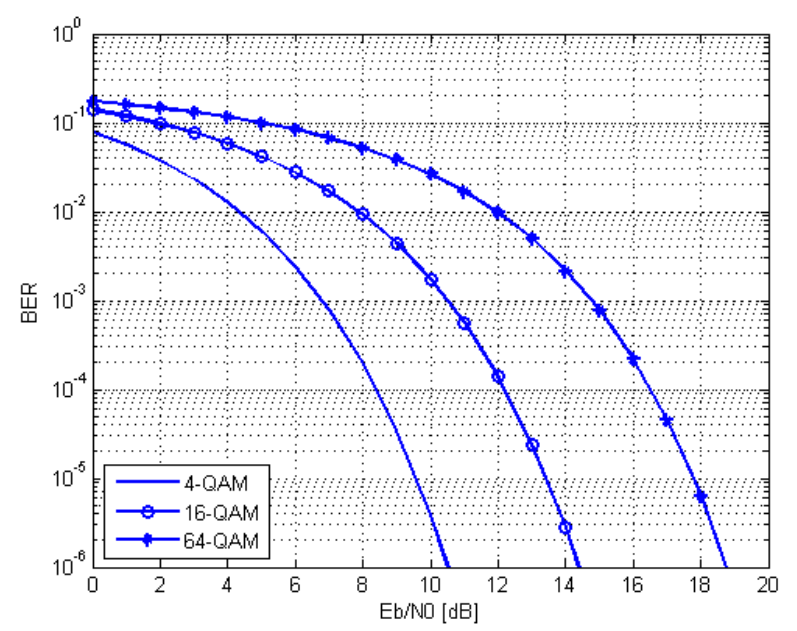

Fig. 7 Out-of-service measurable waterfall $B E R\left(E_{\mathrm{b}} / N_{0}\right)$ curves, where $E_{\mathrm{b}}$ and $N_{0}$ are energy of a bit and noise spectral density, respectively [18]

However, in practical measurements with digital transmission systems, the eye diagram in-phase (I) and quadrature-phase $(\mathrm{Q})$ closures - $I_{\mathrm{CLO}}$ and $Q_{\mathrm{CLO}}$, respectively, are used to express a general overview of a condition of a radio system, primarily the margin against noise and other impairments, represented by the percentage of modulation symbols separation in I and Q directions, respectively [18].

I and $\mathrm{Q}$ eye diagrams can be obtained by means of a simple dual-beam oscilloscope, by synchronizing its time base with data clock, Fig. 8, and setting up infinite screen persistence with analog scopes, or cumulative data acquisition mode, with digital ones, Fig. 9. 


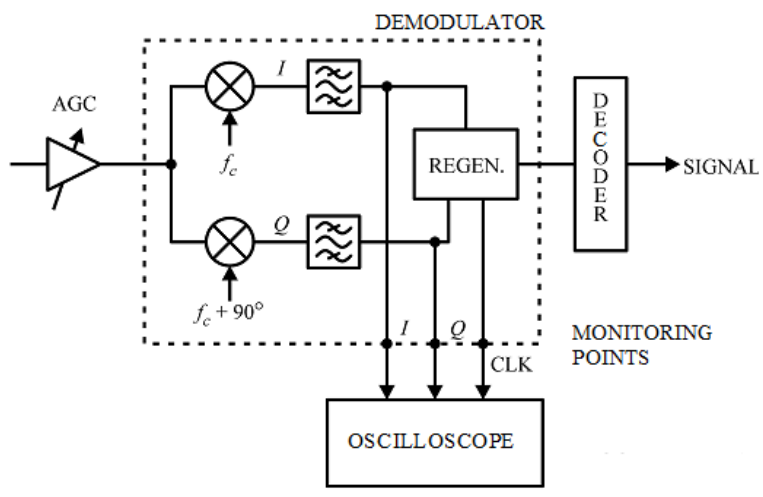

Fig. 8. Measuring eye diagrams with a dual-beam oscilloscope [1]

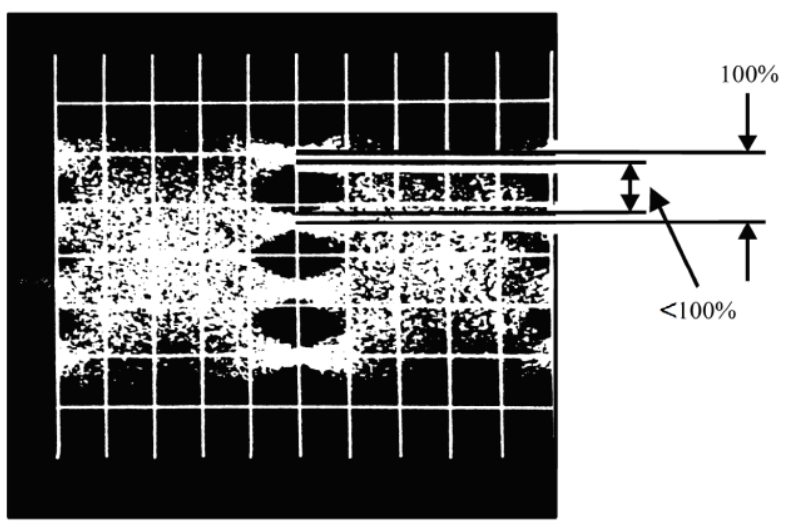

Fig. 9. Practical eye diagram (the percentage denotes eye openess, i.e. the complement of the noise margin) [1]

So, to estimate $\operatorname{BER}\left(E_{\mathrm{b}} / N_{0}\right)$ in (10), we need to calculate $E_{\mathrm{b}} / N_{0}$ from closure. It has been shown that the closure is related to $C / N$ as it follows [18]:

$$
\frac{C}{N}=20 \log \left(\frac{100 \cdot C_{1}}{\sqrt{I_{\mathrm{CLO}}^{2}+Q_{\mathrm{CLO}}^{2}}}\right)
$$

where the constant $C_{1}$ represents the rms power of the carrier, and depends on the actual modulation scheme (e.g. $\sqrt{10}$ for 16 QAM).

Furthermore, as:

$$
\frac{E_{b}}{N_{0}}=\frac{C}{N} \cdot B_{\mathrm{N}} \cdot T
$$

then, taking into account (11), (12) can be rewritten as:

$$
\frac{E_{b}}{N_{0}}=20 \log \left(\frac{100 \cdot C_{1}}{\sqrt{I_{\mathrm{CLO}}^{2}+Q_{\mathrm{CLO}}^{2}}}\right) \cdot B_{\mathrm{N}} \cdot T
$$

For the LTE receiver noise bandwidth of $B_{\mathrm{N}}=1.25 \mathrm{MHz}$, (13) can be presented in tabular form, which, for 16 QAM modulation, is Table 1 .
TABLE I

EQUIVALENT $E_{\mathrm{B}} / N_{0}$ AS A FUNCTION OF I AND Q CLOSURES FOR 16 QAM [18]

\begin{tabular}{|c|c|c|c|c|c|c|c|}
\hline Q I & 10 & 11 & 12 & 13 & 14 & 15 & 16 \\
\hline 10 & 14.0 & 13.7 & 13.3 & 13.0 & 12.6 & 12.3 & 11.9 \\
\hline 11 & & 13.3 & 13.0 & 12.7 & 12.4 & 12.0 & 11.7 \\
\hline 12 & & & 12.7 & 12.4 & 12.1 & 11.8 & 11.5 \\
\hline 13 & & & & 12.1 & 11.8 & 11.5 & 11.2 \\
\hline 14 & & & & & 11.5 & 11.3 & 11.0 \\
\hline 15 & & & & & & 11.0 & 10.7 \\
\hline 16 & & & & & & & 10.5 \\
\hline
\end{tabular}

This way, the closure values in (13) or Table 1 can be substituted into (9).

So, e.g. for 16 QAM, where $M=16$, substituting (13) into (10), provides $B E R$ as it follows:

$$
B E R=Q\left[4 \cdot \sqrt{\log \left(\frac{100 \cdot \sqrt{10}}{\sqrt{I_{\mathrm{CLO}}^{2}+Q_{\mathrm{CLO}}^{2}}}\right) \cdot B_{\mathrm{N}} \cdot T}\right]
$$

Finally, for complete in-service PAPR estimation, (14) has to be substituted into (5).

\section{VERIFICATION}

In order to verify (5), it was necessary to conduct Monte Carlo (MC) simulations of various symbols belonging to the three LTE-relevant modulation types: 4-QAM, 16-QAM and 64-QAM, and perform uncoded BER tests without time dispersion (equivalent to the perfect $\mathrm{CP}$-protected channel), and with high SNR [1].

\section{A. Verification of PAPR Estimation}

With back-off values ranging from 0 to $12 \mathrm{~dB}$, the HPA non-linearity was introduced just to the maximal-amplitude outer symbols in the constellation, so reducing the PAPR and enabling bit error occurrences to be counted for the short-term $B E R$ calculation and checking (5).

In the simplified PAPR model, we considered that without normalization, the I and Q component of the maximal 4, 16 and 64-QAM modulation symbols' amplitudes are equal to 1, 3 and 7, respectively. The normalization is performed by dividing the above values by $\sqrt{2}, \sqrt{10}$ and $\sqrt{42}$, respectively, so enabling the unit mean symbol energy [4], i.e.:

$$
\mathrm{E}\left[s_{k}^{2}\right]=1
$$

This implies:

$$
\begin{aligned}
& s_{k, \max 4-\mathrm{QAM}}^{2}=\frac{1}{2} \cdot\left(1^{2}+1^{2}\right)=1 \\
& s_{k, \max 16-\mathrm{QAM}}^{2}=\frac{1}{10} \cdot\left(3^{2}+3^{2}\right)=1.8: \quad 1 \leq k \leq M \\
& s_{k, \max 64-\mathrm{QAM}}^{2}=\frac{1}{42} \cdot\left(7^{2}+7^{2}\right) \approx 2.33
\end{aligned}
$$


The instantaneous (per-OFDM-symbol) PAPR, defined as [4]:

$$
P A P R_{k} \approx \frac{P_{\text {peak }}}{P_{\text {avg }}}=\frac{\max _{m}\left\{\frac{1}{2} \cdot \sum_{m=1}^{M} s_{m}^{2}\right\}}{\frac{1}{2} \cdot \sum_{m=1}^{M} s_{m}^{2}}=\frac{\max _{m}\left\{\sum_{m=1}^{M} s_{m}^{2}\right\}}{\sum_{m=1}^{M} s_{m}^{2}}
$$

can be regarded as determined by the maximal amplitude of the 64-QAM symbols only, providing the highest PAPR values:

$$
P A P R_{k \text { absmax }}=\frac{M \cdot s_{k, \max 64-\mathrm{QAM}}^{2}}{\sum_{m=1}^{M} s_{m}^{2}} ; 1 \leq k \leq M
$$

On the other hand, more realistic PAPR values are obtained by accordingly modifying (17) to take into account not only the (rarely appearing) 64-QAM highest-amplitude symbols, but all maximal amplitudes' values from (16), weighted by their probabilities of occurrence in the OFDM symbol, which can take various values, such as e.g. [19]:

$$
\begin{aligned}
& P\left(s_{k} \in 4-\mathrm{QAM}\right)=0.4 \\
& P\left(s_{k} \in 16-\mathrm{QAM}\right)=0.5 ; \quad 1 \leq k \leq M \\
& P\left(s_{k} \in 64-\mathrm{QAM}\right)=0.1
\end{aligned}
$$

Accordingly, the corresponding PAPR plots for the three LTE-relevant modulations, and back-off values of 5, 10 and $15 \mathrm{~dB}$, are presented in Figures 10, 11 and 12, respectively.

Observed intra-figures, the below plots reflect dispersion among the modulation types for the same $P A P R$ value, where the higher modulation order implies the larger $B E R$. Moreover, observed inter-figures, it is obvious that for any modulation algorithm, the larger back-off values increase the corresponding tolerable $P A P R$ values for the same $B E R$ value.

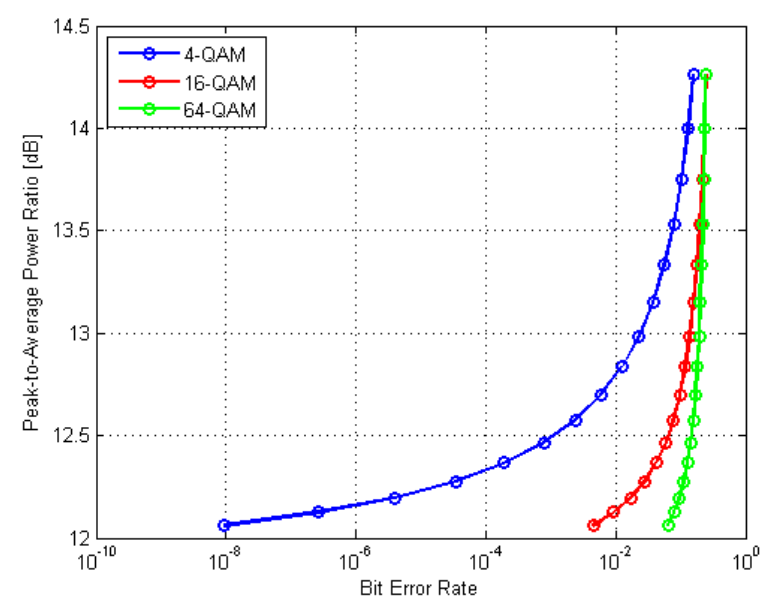

Fig. 10. PAPR vs BER with 5 dB HPA back-off

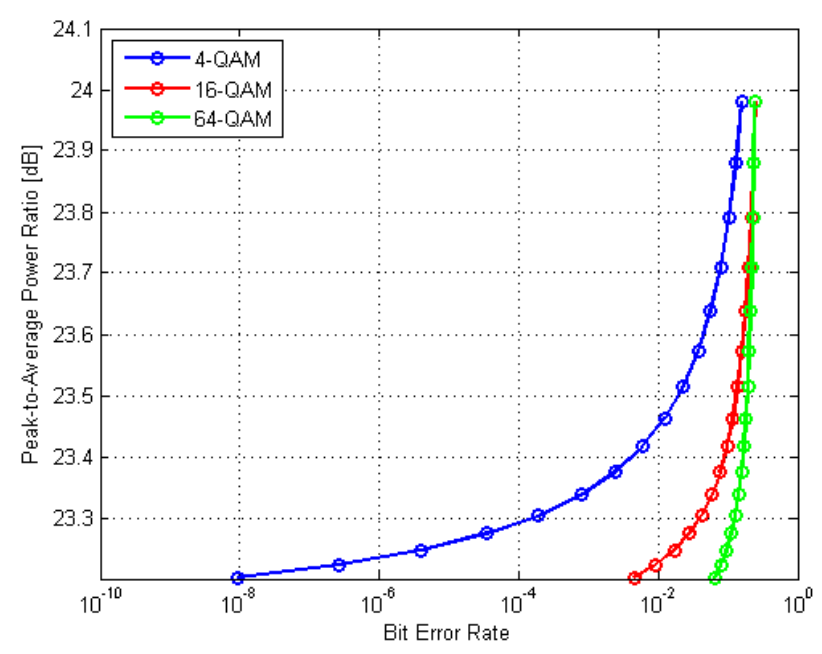

Fig. 11. PAPR vs BER with $10 \mathrm{~dB}$ HPA back-off

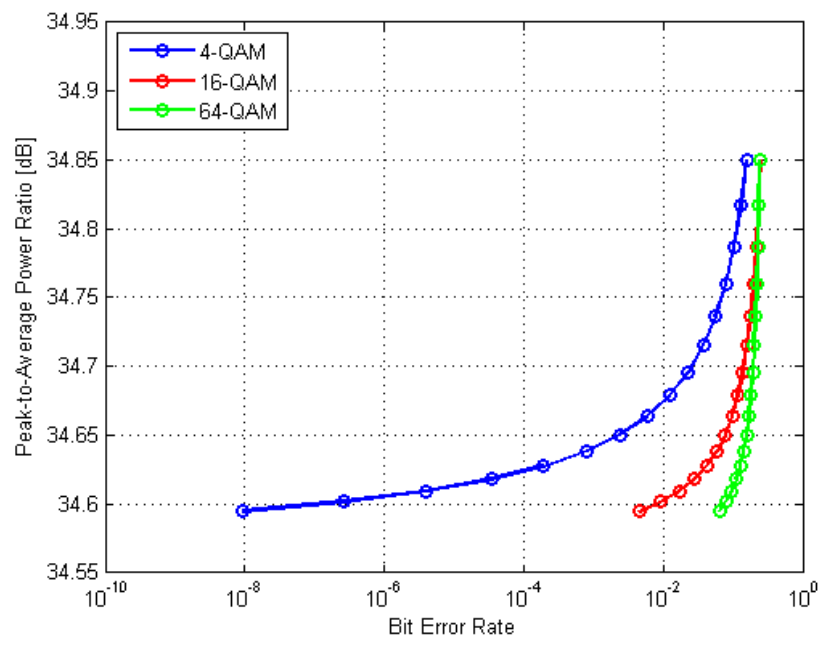

Fig. 12. PAPR vs $B E R$ with $15 \mathrm{~dB}$ HPA back-off

Finally, in Table II the exemplar PAPR vs BER relationship with $5 \mathrm{~dB}$ back-off for 4-QAM, 16-QAM and 64-QAM modulation schemes, are presented, as coming out of the estimation (5) and MC simulations.

As it can be seen from the table, excellent matching between the estimated and the MC-simulated $P A P R$ values is found (with the tolerance to PAPR decreasing with the order of $\mathrm{QAM}$ ), which verifies the model under the presumed conditions.

\section{B. Verification of Estimation of $C F O$-induced Phase Deviation}

Furthermore, the corresponding phase deviation plots for the three LTE-relevant modulations, and the CFO values of 5, 70 and $300 \mathrm{~Hz}$, are presented in Figures 13, 14 and 15, respectively. 


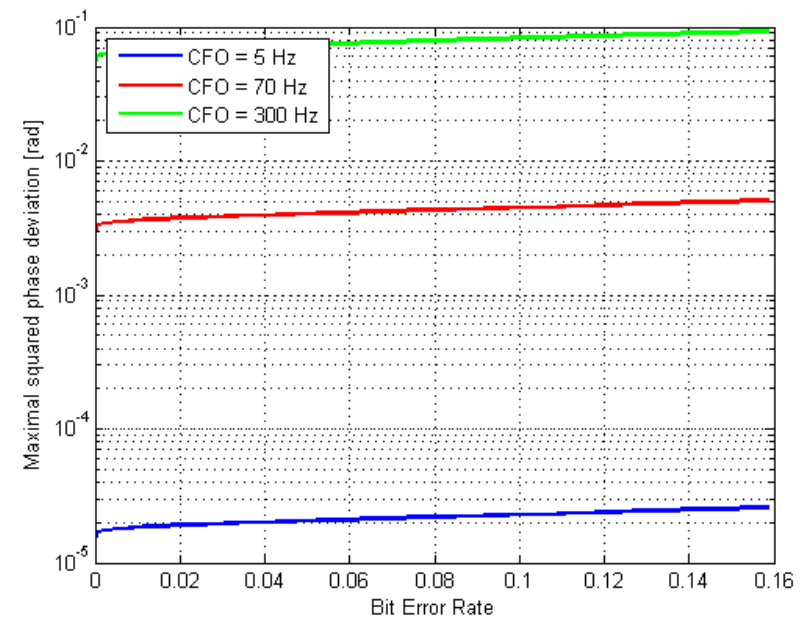

Fig. 13. $\Delta \Phi_{k \max }^{2}$ vs BER with 5 dB HPA back-off, 4 QAM

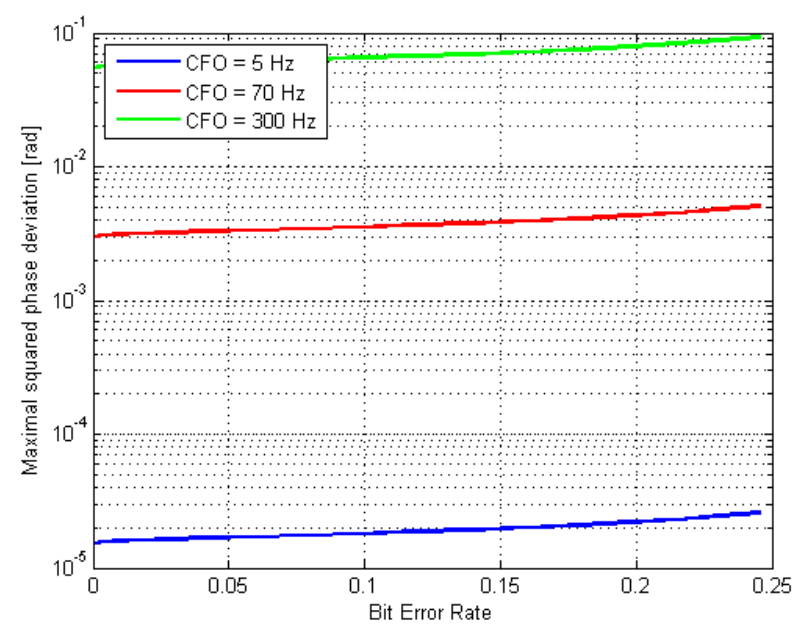

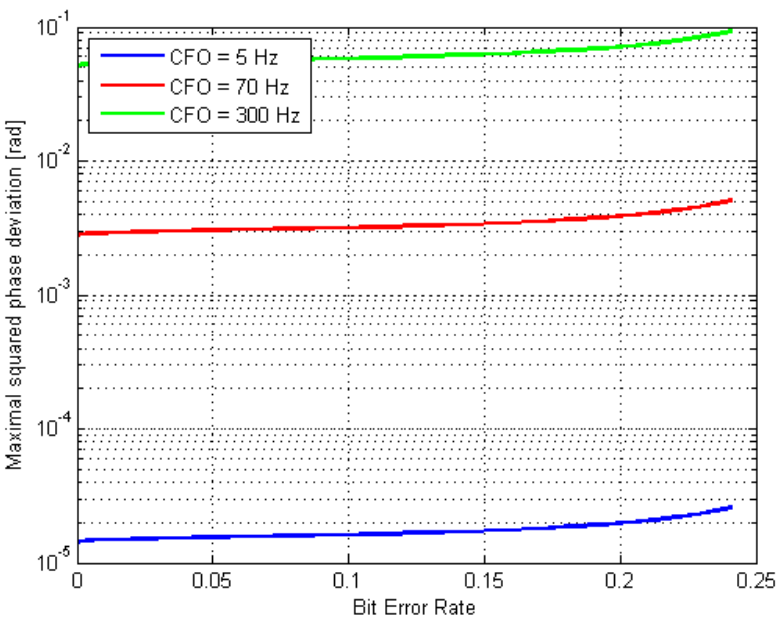

Fig. 15. $\Delta \Phi_{k \max }^{2}$ vs $B E R$ with 5 dB HPA back-off, 64 QAM

If compared intra-figures, the above plots reflect dispersion among the CFO values for the same modulation type, where the higher CFO implies the larger BER. Moreover, compared inter-figures, the higher modulation order is less tolerable to CFO for the same $B E R$ value.

Finally, in Table III, the exemplar $\Delta \Phi_{k \max }^{2}$ vs $B E R$ relationship with $5 \mathrm{~dB}$ back-off and $300 \mathrm{~Hz}$ CFO, for 4-QAM, 16-QAM and 64-QAM modulation schemes, are presented, as coming out of the estimation (5) and MC simulations.

The table exhibits excellent matching found between the estimated and the MC-simulated BER values, with the tolerance to CFO phase deviation decreasing with the order of QAM. This verifies the model under the presumed conditions.

Fig. 14. $\Delta \Phi_{k \max }^{2}$ vs BER with 5 dB HPA back-off, 16 QAM

TABLE II

PAPR vs. BER; 4-QAM

\begin{tabular}{|c|c|c|c|c|c|c|}
\hline & \multicolumn{3}{|c|}{ Estimation (5) } & \multicolumn{3}{c|}{ MC simulations } \\
\cline { 2 - 7 } & 4-QAM & 16 -QAM & 64-QAM & 4-QAM & 16-QAM & 64-QAM \\
\hline$B E R$ & $10^{-3}$ & $1.27 \cdot 10^{-2}$ & $1.53 \cdot 10^{-2}$ & $1.12 \cdot 10^{-3}$ & $1.36 \cdot 10^{-2}$ & $1.58 \cdot 10^{-2}$ \\
\hline$P A P R[\mathrm{~dB}]$ & 12.50 & 12.50 & 12.50 & 12.50 & 12.50 & 12.50 \\
\hline
\end{tabular}

TABLE III

CFO PHASE DEVIATION vs. BER; 4-QAM

\begin{tabular}{|c|c|c|c|c|c|c|}
\hline & \multicolumn{3}{|c|}{ Estimation (5) } & \multicolumn{3}{c|}{ MC simulations } \\
\cline { 2 - 7 } & 4-QAM & $16-Q A M$ & $64-Q A M$ & 4-QAM & 16-QAM & 64-QAM \\
\hline$B E R$ & $10^{-3}$ & $1.27 \cdot 10^{-2}$ & $1.53 \cdot 10^{-2}$ & $1.12 \cdot 10^{-3}$ & $1.36 \cdot 10^{-2}$ & $1.58 \cdot 10^{-2}$ \\
\hline$\Delta \Phi_{k \max }^{2}[\mathrm{rad}]$ & 0.062 & 0.062 & 0.062 & 0.062 & 0.062 & 0.062 \\
\hline
\end{tabular}




\section{CONCLUSION}

In many practical situations, specifically with OFDM transmission over LTE-Advanced downlink, applying carrier aggregation, $P A P R$ values can be excessive, so quick and simple $P A P R$ estimation can be of interest. Therefore, in this paper the link abstraction principle is applied on $B E R$ that is considered to be determined just by the HPA nonlinear distortion, i.e. that errors occur just due to HPA nonlinearity and the inadequate $P A P R$ vs back-off relationship.

Moreover, by capitalizing on its relationship with $P A P R$, the other dominant OFDM-inherent impairment - the CFO-caused phase deviation in particular, can also be estimated by means of common BER testing.

However, as network operators consider out-of-service $B E R$ testing not preferable, under such circumstances, we propose simple in-service $B E R$ estimation from I and Q eye diagrams, and then using the obtained value for the so achieved complete in-service $P A P R$ estimation.

The proposed algorithm for estimating both $P A P R$ and CFOinduced phase deviation from $B E R$, is verified by its excellent matching with the corresponding MC-simulated scenario.

\section{REFERENCES}

[1] A. Lipovac, A. Mihaljević," BER Based OFDM PAPR Estimation", Proc. 26th International Conference on Software, Telecommunications and Computer Networks - SoftCOM, pp.1-6, Split-Supetar, 2018. DOI: 10.23919/SOFTCOM.2018.8555780

[2] A. Lipovac, V. Lipovac and B. Modlic, "Modeling OFDM Irreducible BER with Impact of CP Length and CFO in Multipath Channel with Small Delay Dispersion", Wireless Communications and Mobile Computing, vol. 16, no. 9, pp. 1065-1077, 2016. DOI: $10.1002 / \mathrm{wcm} .2586$

[3] M. Rumnay, LTE and the Evolution of 4G Wireless; Design and Measurements Challenges, 2nd edition, John Wiley \& Sons, 2013.

[4] A. Molisch, Wireless Communications, 2nd ed., New York, Wiley, 2011.

[5] L. Korowajczuk, LTE,WiMAX and WLAN Network Design, Optimization and Performance Analysis. Wiley 2011.

[6] R. Prasad, OFDM for Wireless Communications Systems, Artech House, Boston, Mass., 2004.

[7] A. A. Hassan, I. S. Ahmed, and S. H. Hossam, "Bit Error Rate Performance of Orthogonal Frequency-Division Multiplexing Relaying Systems with High Power Amplifiers and Doppler Effects“, Wireless Communications and Mobile Computing, vol. 13, no. 8, pp. 734-744, 2013. DOI: $10.1002 / \mathrm{wcm} .1135$

[8] T. Jiang and Y. Wu, "An overview: Peak-to-average power ratio reduction techniques for OFDM signals", IEEE Trans. on Broadcasting, vol. 54, no. 2, pp. 257-268, 2008. DOI: 10.1109/TBC.2008.915770

[9] S. H. Han and J. H. Lee, "An Overview of Peak-to-Average Power Ratio Reduction Techniques for Multicarrier Transmission", IEEE Wireless Communications, vol. 12, issue 2, pp. 56-65, 2005. DOI: 10.1109/MWC.2005.1421929

[10] D. Guel and J. Palicot "Analysis and Comparison of Clipping Techniques for OFDM Peak-To-Average Power Ration reduction", IEEE Transactions on Broadcasting, vol 53, no. 3, pp 719-724, 2007.

[11] H. Ochiai and H. Imai, "Performance analysis of deliberately clipped OFDM signals", IEEE Trans. on Communications, vol. 50, no. 1, pp. 89101, 2002. DOI: $10.1109 / 26.975762$

[12] S. B. Slimane, "Reducing the Peak-to-Average Power Ratio of OFDM Signals Through Precoding", IEEE Transactions on Vehicular Technology, vol. 56, issue 2, pp. $686-695$, 2007. DOI: 10.1109/TVT.2007.891409

[13] S. Y. Le Goff, S. S. Al-Samahi, B. K. Khoo, C. C. Tsimenidis,and B. S. Sharif, "Selected Mapping without Side Information for PAPR Reduction in OFDM", IEEE Transactions on Wireless Communications, vol. 8, no. 7, pp. 3320-3325, 2009. DOI: 10.1109/TWC.2009.070463
[14] T. G. Pratt, N. Jones, L. Smee, and M. Torrey, "OFDM Link Performance with Companding for PAPR Reduction in the Presence of Nonlinear Amplification”, IEEE Transactions on Broadcasting, vol. 52, issue. 2, pp. 261-267, 2006. DOI: 10.1109/TBC.2006.875613

[15] T. Jiang, W. Xiang, P. C. Richardson, J. Guo, and G. Zhu, "PAPR Reduction of OFDM Signals Using Partial Transmit Sequences With Low Computational Complexity", IEEE Transactions on Broadcasting, vol. 53, no. 3, pp. 719-724, 2007. DOI: 10.1109/TBC.2007.899345

[16] H. A. Mahmoud and H. Arslan, "Error Vector Magnitude to SNR Conversion for Nondata-Aided Receivers", IEEE Trans. on Wireless Communications, vol.8, issue.5, pp.2694-2704, May 2009. DOI: 10.1109/TWC.2009.080862

[17] A. Lipovac, B. Modlic, and M. Grgić,"OFDM Error Floor Based EVM Estimation", Proc. 24rd International Conference on Software, Telecommunications and Computer Networks - SoftCOM, pp.1-5, Split, 2016. DOI: $10.1109 /$ SOFTCOM.2016.7772163

[18] A. Lipovac, V. Lipovac, and M. Hamza, "Verification of OFDM Error Floor Prediction in Time-Dispersive LTE FDD DL Channel", Wireless Personal Communications, vol. 93, no. 3, pp. 853-875, 2017. DOI: 10.1007/s11277-014-2232-y

[19] A. Lipovac, S. Isak-Zatega, and P. Njemčević," In-service Testing OFDM Error Floor by Constellation Analysis", Proc. 25th International Conference on Software, Telecommunications and Computer Networks SoftCOM, pp.1-6, Split, 2017. DOI: 10.23919/SOFTCOM.2017.8115549

[20] J. C. Ikuno, C. Mehlführer and M. Rupp, "A Novel Link Error Prediction Model for OFDM Systems with HARQ", Proc. 2011 IEEE International Conference on Communications (ICC), pp. 1-5, Kyoto, 2011. DOI: $10.1109 /$ icc.2011.5962464

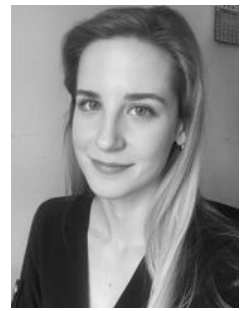

Adriana Lipovac took her Bachelor degree from the University of Sarajevo, in 2010, and her Master degrees in both Electrical Engineering and in Computing from the University of Dubrovnik, in 2012, when she also received Rector's award as the best student in her generation. Following graduation, she continued her work in the field of mobile communications as a teaching assistant at the University of Dubrovnik, while conducting her research on modelling $4 \mathrm{G}$ wireless systems physical layer performance, at the University of Zagreb, where she received her Ph.D. in 2014. Since 2015, she has been working as an assistant professor at the Department of Electrical Engineering and Computing, University of Dubrovnik, where she also holds the position of Head of the Laboratory for Communications Technology. She was the local organizing co-chair for the International Wireless Communications and Mobile Computing Conference IWCMC and the Global Wireless Summit GWS, in 2015 and 2016, respectively. During and after her OFDM symbols transfer performance on the radio channel based research, she published several scientific papers in relevant journals, cited in WoS databases, as well as in the proceedings of international scientific conferences.

Ante Mihaljević received his Bachelor's and Master's degrees in Marine Electrical Engineering and Communication Technologies from the University of Dubrovnik, Croatia, in 2016 and 2018, respectively. Currently he is a teaching assistant at the Department of Electrical Engineering and Computing, University of Dubrovnik. He is also a $\mathrm{PhD}$ student at the Faculty of Electrical Engineering and Computing, University of Zagreb, Croatia. 